Military Technical College

Kobry El-Kobbah,

Cairo, Egypt.

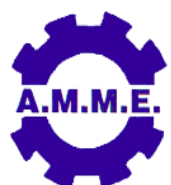

$17^{\text {th }}$ International Conference

on Applied Mechanics and

Mechanical Engineering.

\title{
MODELING OF SUSPENSION SYSTEM OF A LIGHT TRACKED VEHICLE
}

\author{
M. M. Abdelaziz*, A. M. Salem* and W. G. Kotb*
}

\begin{abstract}
Tracked vehicle suspension has to isolate a vehicle hull from road irregularities in order to maximize vehicle speed, preserve its systems and maintain combat activities. The mobility performance of tracked vehicles is often limited by the operator's endurance to withstand the transmitted shocks and vibrations and his ability to maintain control. The maximum allowable vehicle speed varies with the roughness of a particular terrain and is primarily influenced by the suspension system design. The influence of the shock absorber characteristics of armourd fighting vehicle BMP-1 on vehicle dynamics in vertical plane was analyzed. This is performed by realizing a mathematical model for the vehicle suspension using MATLAB/SIMULINK in order to obtain the vibrational response of the vehicle. The model has 8 DOF including the response of the bounce and pitch for vehicle hull and six road wheels. The nonlinear characteristics of the vehicle shock absorber obtained from experimental measurement were fitted to the model to obtain accurate results. The shock absorber was tested with an excitation frequency range from $1 \mathrm{~Hz}$ to $5 \mathrm{~Hz}$, corresponding to typical velocities of vehicle generated by road irregularities. The model is designed for different road shapes with different amplitudes and frequencies.
\end{abstract}

\section{KEYWORDS}

Suspension, shock absorber, tracked vehicle, vibration.

\footnotetext{
* Egyptian Armed Forces.
} 


\section{INTRODUCTION}

Preserving drive smoothness is one of the basic requirements imposed on tracked vehicles operation and it depends on design and quality of vehicle suspension. The ability of military vehicles to cross all types of terrain depends heavily on their suspension systems. The higher is the speed of the tracked vehicle, the higher is the vibration induced by rough road conditions. These vibrations have passive effect on vehicle combat activities such as firing accuracy on move and observation of battle field; also they have bad effect on crew comfortability and on vehicle systems and aggregates. Excessive vibration limits the vehicle maximum speed and consequently it reduces survivability and mobility and accordingly reduces the operational efficiency in combat situations. Accordingly, the type and quality of suspension system play an important role on the dynamic performance of the vehicle. In general, tracked vehicle suspensions comprises multiple, independent road wheels that are mounted on axle arms. The suspension system also incorporates elastic elements and shock absorbers. During tracked vehicle motion, shocks are generated due to road roughness and transmitted through tracks to the road wheels causing deformation of the elastic elements which results in vibration of hull. The hull vibration is damped by the shock absorbers. The shape and nature of hull vibration depend on whether the suspension has linear or nonlinear characteristics.

A testing and analysis methodology using the MTS 831 elastomer characterization machine has been used to obtain the dynamic properties of automotive shock absorber with different types of input excitation such as stepped sine sweep excitation, sine on sine testing using two different sine waves and random excitation taking into account the temperature and stroke length [1]. The analysis shows that the output force decreases as temperature increases and the stroke length of the shock absorber affects its parameters. Moreover, hydraulic test stand is used to identify the characteristics of shock absorber. The damping force and motion of the damper that had been measured in quarter car model in real time is used to show that the damping forces of the shock absorbers depend mainly on the velocities of the moving pistons and are not correlated to the displacement of the piston [2-5]. The tracked vehicles vibration response in dependence with suspension system parameters such as torsion bar rigidity and damping coefficient of shock absorber are introduced in [6].

In previous studies, the influence of the suspension performance on the vehicle ride comfort has been investigated through comprehensive analyses of suspension design parameters using computer simulation models. These models are commonly introduced to quantify the effect of the proposed parameters before the final design process and testing. Some of the models have been used to study the tracked vehicle performance with varying complexities [7-9]. Few of these mathematical models have been validated against field tests with some noticeable success [8]. Most of the literature concerning the evaluation of tracked vehicle performance is based on the influence of the torsion bars stiffness and/or assembly angle, the number of road wheels and damping coefficient of shock absorbers on the vehicle dynamic characteristics[10].

The infantry fighting vehicle BMP-1 is selected to study the effect of its suspension parameters on the vehicle vibration through realizing a mathematical half model for the vehicle suspension using MATLAB/SIMULINK in order to obtain the vibrational 
response of the vehicle. The model has $8 \mathrm{DOF}$ including the response of the bounce and pitch for vehicle hull and the vertical displacement of six road wheels. The nonlinear characteristics of the vehicle shock absorber obtained from experimental measurement by using the MTS machine are fitted to the model to obtain accurate results. The shock absorber is tested with an excitation frequency range from $1 \mathrm{~Hz}$ to $5 \mathrm{~Hz}$ corresponding to typical velocities of vehicle generated by road irregularities. The model is designed for different road shape inputs such as sine wave excitation input, random input with different amplitudes and frequencies.

\section{SUSPENSION SYSTEM OF THE BMP-1 TRACKED VEHICLE}

The suspension system of the BMP-1 tracked vehicle is a conventional suspension. Each side of the vehicle is equipped with six road wheels, six torsion bars and buffers, and two shock absorbers. The shock absorbers are fixed at the extreme road wheels to provide an effective damping of vehicle vibration. The maximum speed of the BMP1 tracked vehicle reaches $65 \mathrm{~km} / \mathrm{hr}$ and off road speed ranges from 35 to $45 \mathrm{~km} / \mathrm{hr}$. This relatively high speed causes bad effect on the crew and vehicle aggregates especially when the vehicle moves on rough terrains. One of the most important elements of the suspension system is the shock absorber as it produces the damping force required to decrease vehicle vibration. The components of the suspension system are shown in figure 1.

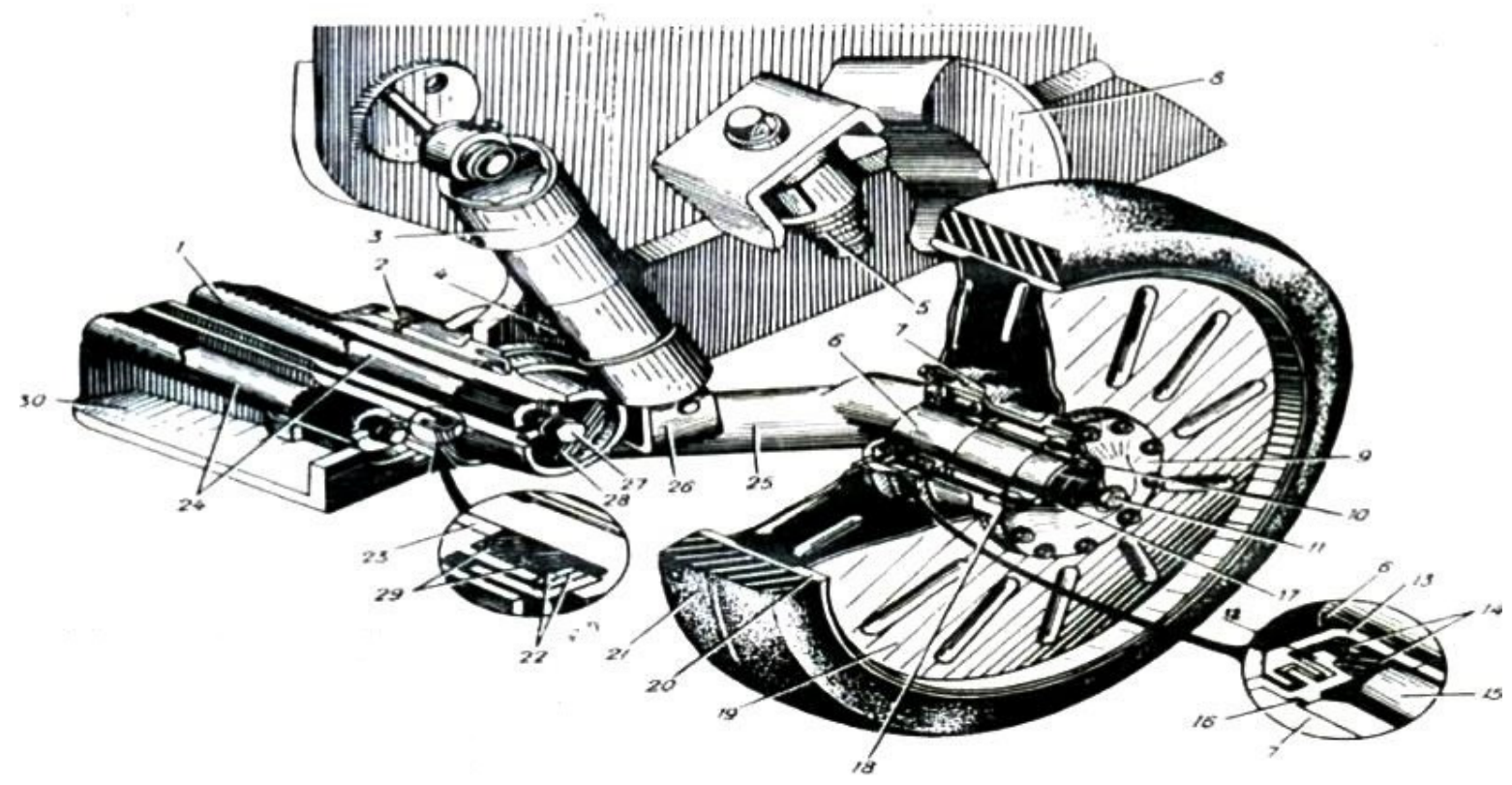

1-Road wheel arm spindle, 2-Suspension bracket, 3-Hydraulic shock absorber, 4-Plug, 5-Road wheel arm spring stop,6-Road wheel spindle, 7-Hub, 8-Stop, 9-Cap, 10-Retainer, 11-Filler plug,12-Labyrinth seal, 13-Labyrinth seal cover,14-Cup, 15-Roller bearing, 16-packing ring, 17-locking nut, 18-Rubber ring,19-Disk, 20-Rim, 21-Rubber tire, 22-Shims, 23-Bussing, 24-Torsion bars,25-Road wheel arm, 26Yoke, 27-Nuts, 28-Cover, 29-Cup, 30-Hull bottom

Figure 1: Components of BMP-1 suspension system unit. 


\section{ROAD EXCITATION PROFILES}

During vehicle motion on various kinds of terrain such as sinusoidal, ramp, random and step terrains, it is subjected to different excitation forces. Consequently, it is of rather important to study and know the effect of these excitation forces on the vibrational response of vehicle. The vibrational response of BMP-1 is evaluated in two types of road excitaion profiles which are sinusoidal and random profiles. Figure 2 demonstrates typical sinusoidal and random road profiles. The sinusoidal road profile is of wave length $6 \mathrm{~m}$ and amplitude $0.1 \mathrm{~m}$ [11] while the random road profile is expressed by a describing equation relates the amplitude of excitation and its derivatives to the road irregularities and vehicle speed [12].

$$
\dot{z}_{r}+\rho v z_{r}=v w_{n}
$$

where, $z$ is road amplitude, $v$ is vehicle speed, $w_{n}=2 \sigma^{2} \rho v$ is white noise intensity, and $\rho$ is road roughness parameter. The roughness parameter is chosen depending upon the road conditions. For this study a road roughness $\rho=0.45$ and covariance of the road $\sigma=300 \mathrm{~mm}^{2}$.
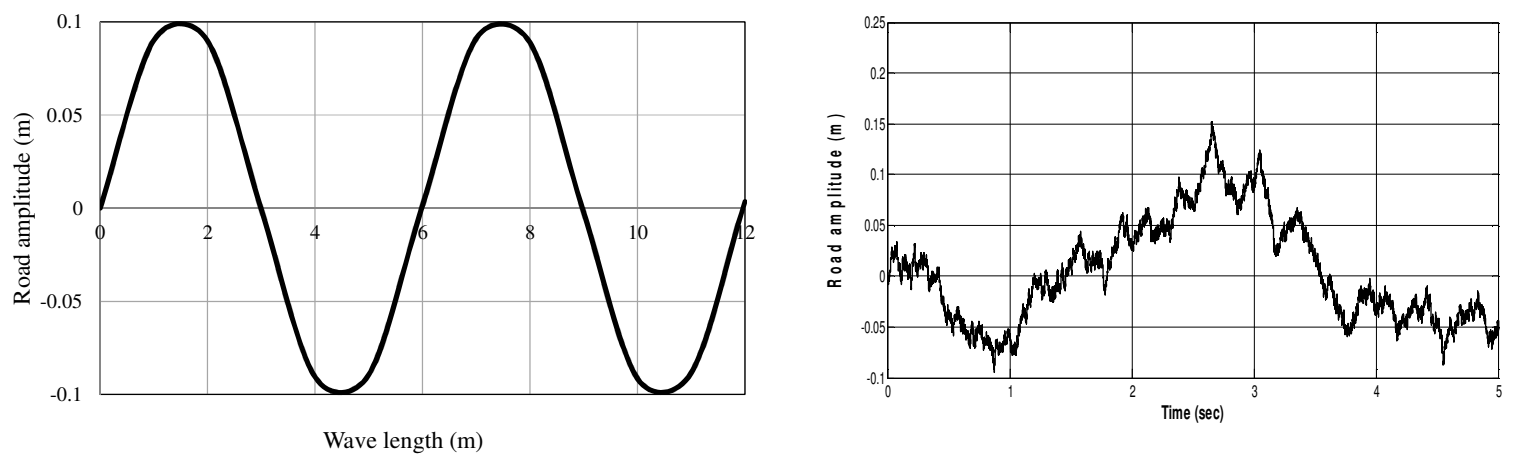

Figure 2: Typical sinusoidal and random road profiles.

\section{EVALUATION OF VEHICLE VIBRATIONAL RESPONSE}

The vibrational response of BMP-1 is evaluated in case of forced excitation resulting from vehicle motion on irregular terrains. This response depends on the quality of suspension system that determined by suspension stiffness and damping coefficient of shock absorber. The suspension stiffness is obtained analytically from vehicle suspension parameters while the damping coefficient is measured experimentally using damper test system. The measured characteristics of the shock absorber are used in half vehicle mathematical model in order to study the response of the vehicle accurately at different road profiles.

\section{Development of a Mathematical Model for BMP-1 Suspension System}

The suspension system of the BMP-1 is represented by a half vehicle model with 8 DOF including the response of vehicle hull in bounce $(Z)$ and pitch $(\varphi)$ as well as six 
road wheels deflection $\mathrm{Z}_{\mathrm{w}}$. The vehicle hull has a sprung mass $\mathrm{m}_{\mathrm{b}}$ and moment of inertia about the lateral axis passing by center of gravity $J_{y}$. The elastic force generated by each road wheel having stiffness $K_{w}$ is denoted by $F_{s p}$. The damping force of such wheels is neglected as it has a small damping value compared with the damping force of the shock absorber $F_{d}$. Figure 3 shows a schematic drawing of vehicle suspension system.

The MATLAB/ SIMULINK model is used to evaluate the dynamic performance of the BMP-1 tracked vehicle with exact (variable) stiffness and damping coefficient at excitation frequency (5) $\mathrm{Hz}$ and amplitude $(0.1) \mathrm{m}$. These parameters correspond to prescribed sinusoidal road profile. The model also has taken into consideration the stiffness of road wheels $k_{w}$ as they have rubber tires frame. Figure 4 shows the block diagram of the proposed Simulink model for the vehicle suspension.

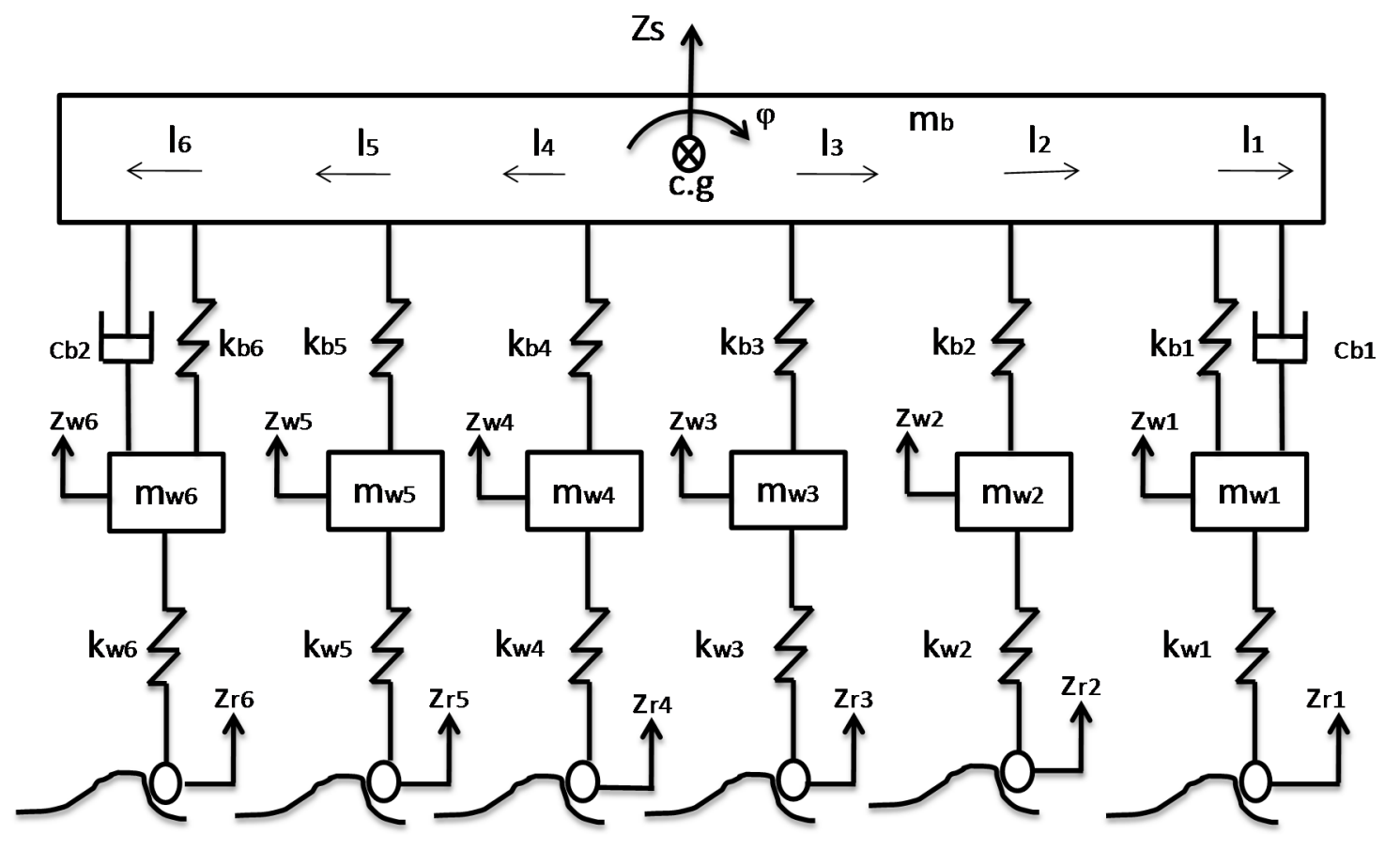

Figure 3: Schematic drawing of vehicle suspension system.

The equations of motion of vehicle hull moving over an irregular terrain in bounce and pitch are expressed by:

Bounce:

$m_{b} Z_{b} \cdot+\sum_{1}^{6} F_{s p i}+\sum_{i=1,6} F_{d i}=0$

$\underline{\text { Pitch }}$

$J_{y} \varphi_{b} \cdot+\sum_{1}^{6} l_{i} F_{s p i}+\sum_{i=1,6} l_{i} F_{d i}=0$ 
While the equation of motion of each road wheel is:

$m_{w} Z_{w i}^{\ddot{*}}-\sum_{1}^{6} F_{s p i}-\sum_{i=1,6} F_{d i}+\sum_{1}^{6} F_{w i}=0$

where

$$
\begin{aligned}
& F_{s p i}=k_{b}\left(z_{b} \pm l_{i} \varphi_{b}-z_{w 1}\right) \\
& F_{d i}=c_{b}\left(z_{b} \pm l_{1} \varphi_{b}-z_{w 1}\right) \\
& F_{w i}=k_{w}\left(z_{w b}-z_{r}\right)
\end{aligned}
$$
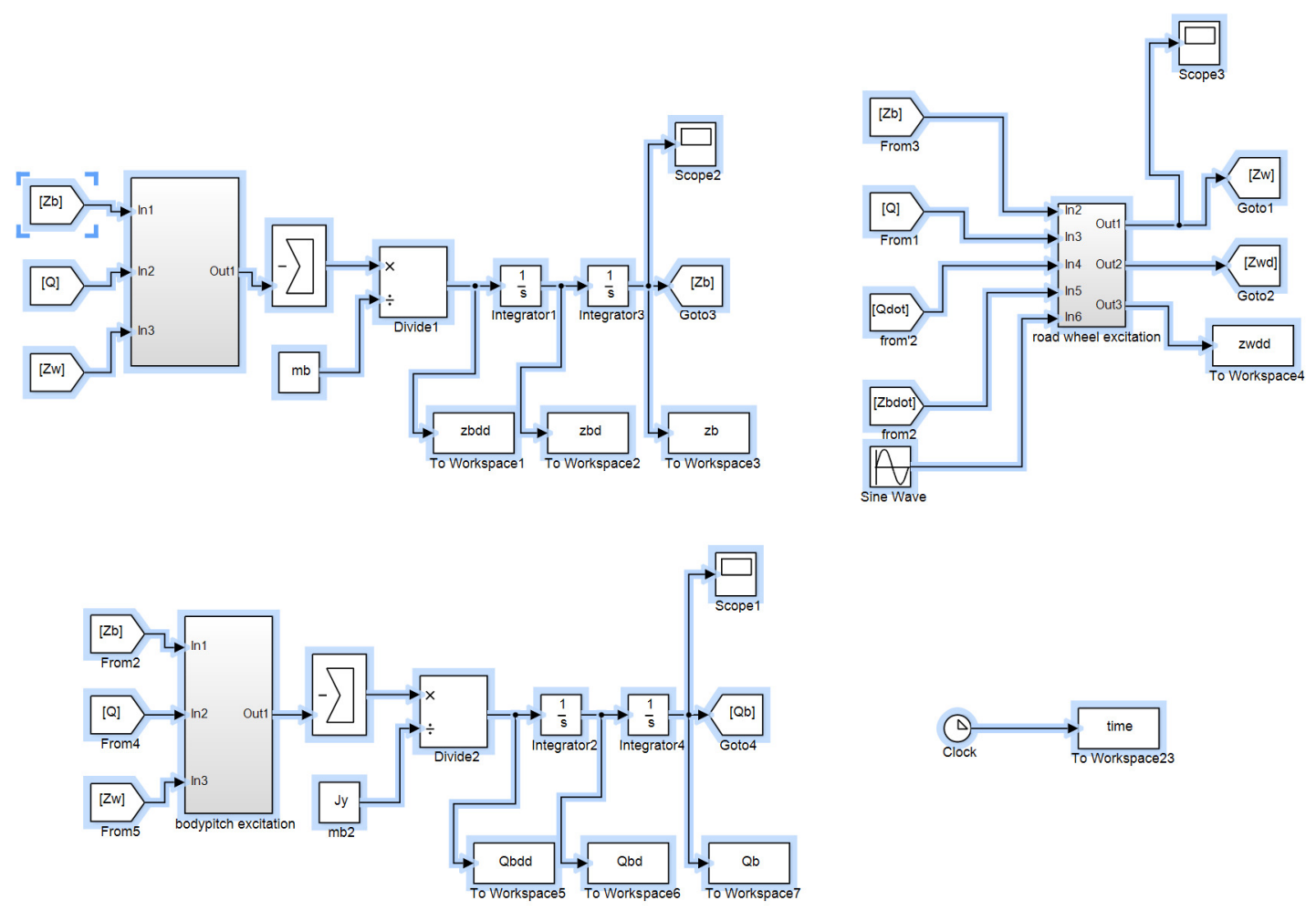

Figure 4: Block diagram of suspension system SIMULINK model.

\section{Suspension Characteristics for BMP-1 Tracked Vehicle}

Suspension characteristics are the relation between the vertical force acting on road wheel and its vertical displacement. The acting force on the road wheel $P_{k}$ can be calculated according to the following relation [6]:

$$
P_{k}=\frac{\pi d^{4} G}{32 L} \beta \frac{\pi}{180} \frac{1}{a_{o} \cos \left(\beta_{o}-\beta\right)}
$$

where $d$ is the diameter of the torsion bar, $L$ is its length, $\beta_{o}$ is its assembly angle $\beta$ is the twist angle, $G$ is the modulus of elasticity in shear, and $a$ is length of the axle arm. 
The vertical displacement of the road wheel $f_{k}$ is calculated by:

$$
f_{k}=a \sin \beta_{o}-a \sin \left(\beta_{o}-\beta\right)
$$

where $a$ is length of the axle arm. For BMP-1 parameters and for different twisting angle $\beta$, the suspension characteristics and the suspension stiffness of the vehicle are shown in figure 5 . It can be found from the figure that the average value of suspension stiffness is about $700 \mathrm{~N} / \mathrm{cm}$. The following parameters are used for calculation the suspension characteristics of BMP-1: $d=3.8 \mathrm{~cm}, L=204 \mathrm{~cm}, \beta_{o}=35^{\circ}, \beta_{\max }=50^{\circ}, a=$ $35 \mathrm{~cm}, G=8 \times 10^{6} \mathrm{~N} / \mathrm{cm}^{2}$

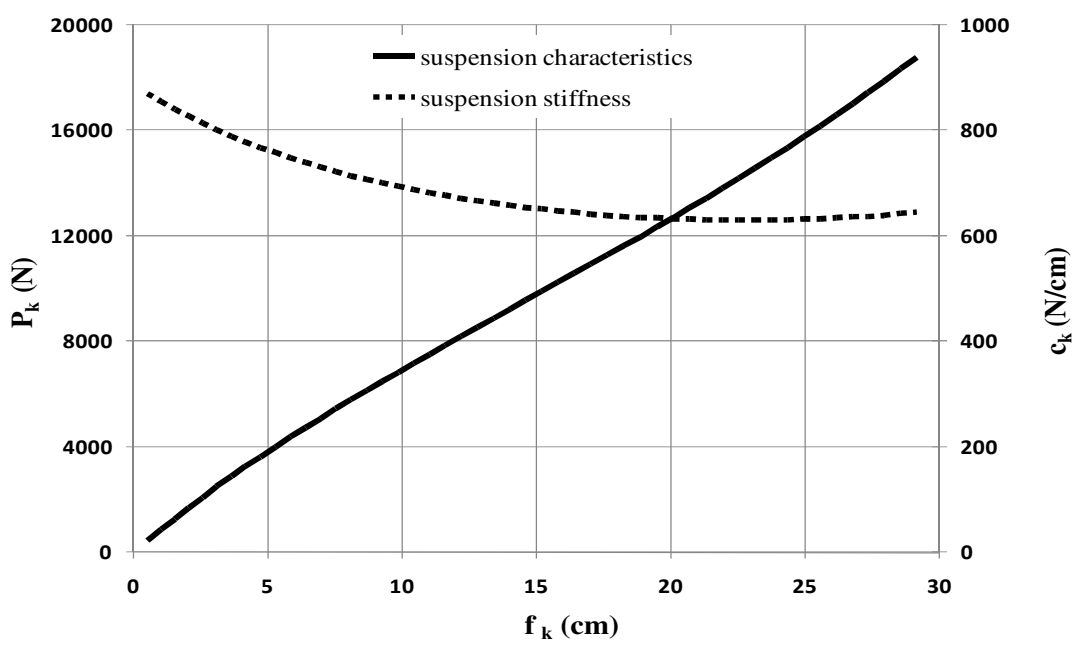

Figure 5: Suspension characteristics of BMP-1.

\section{IDENTIFICATION OF SHOCK ABSORBER DAMPING COEFFICIENT}

The damper test system (MTS 793) used for measuring the dynamic characteristics of the BMP-1conventional damper is shown in figure 6. The system consists of, the loading frame, the control unit and the hydraulic power unit. The loading frame has two vertical columns with adjustable upper crosshead and base (fixed to the frame). On the upper crosshead frame, there is a fixture to clamp the upper end of the damper where the load cell is attached for measuring the generated damping force. The actuator mounted on the lower frame has a built-in linear voltage displacement transducer (LVDT) to measure the displacement of the damper moving end.

The lower crosshead frame has a clamp to fix the lower end of the damper. The control unit is used to adjust the frequencies and amplitudes of the excitation. The unit has two channels to measure the displacement and force signals that can be directed to a computer through a data acquisition card. The stroke of the damper was measured before installation to the machine and a midpoint, which equals to half of the stroke, is marked on the piston rod. This will help to adjust the piston to its mid-point to prevent the damper from damage. The oscillation produced by external forces and moments depend on the shape and dimension of the unevenness of terrain and the speed of the 


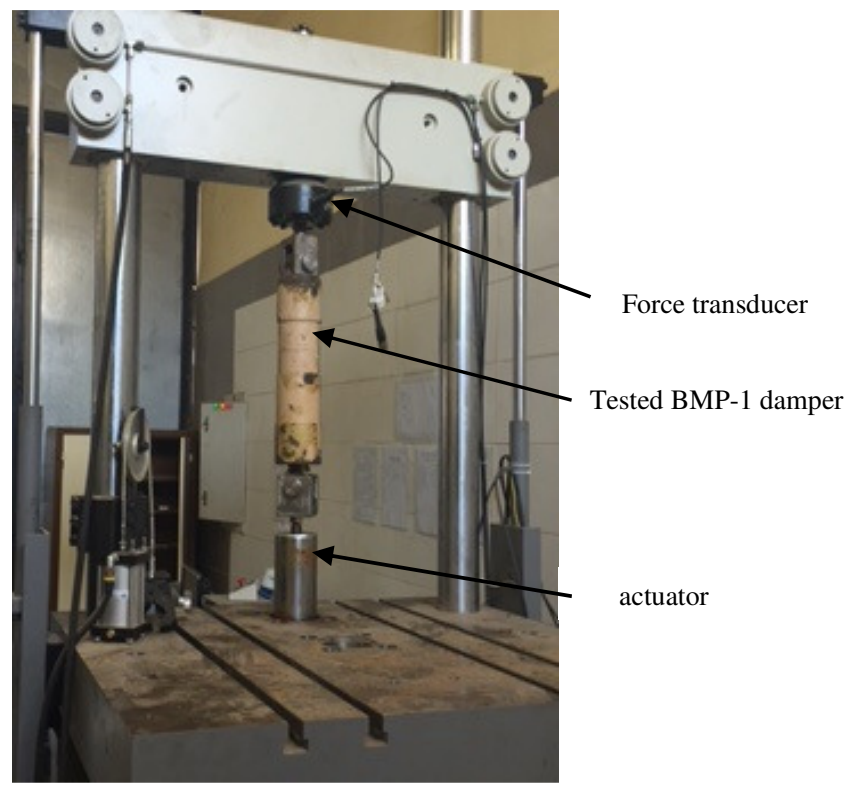

Figure 6: Damper test system used to identify BMP-1 damper characteristics.

vehicle. The excitation frequency of the vehicle, originated from its motion on irregular terrain, depends on the vehicle speed and road profile. Such frequency is calculated from the following relation:

$$
\mathrm{q}=\frac{2 \pi \mathrm{v}}{\mathrm{a}}
$$

where $\mathrm{v}$ is the vehicle speed and $\mathrm{a}$ is the road wave length. For vehicle speed of about $(20-60) \mathrm{km} / \mathrm{hr}$ and for the typical road shown in figure 2 , the excitation frequency is found to be about $1-5 \mathrm{~Hz}$.

The hydraulic shock absorber of the BMP-1 was excited by sinusoidal excitations at a wide range of frequencies and amplitudes. The measured dynamic properties of the shock absorber are presented at excitation frequencies from $1 \mathrm{~Hz}$ to $5 \mathrm{~Hz}$ and at dynamic amplitudes of up to $50 \mathrm{~mm}$. This range represents the loading operational conditions of the damper. Choosing $50 \mathrm{~mm}$ as a maximum amplitude value since the maximum stroke of the actuator rod is restricted to $( \pm 65 \mathrm{~mm})$. The obtained results are corrected for the gas force generated by the gas filled in the shock absorber. Thus, the demonstrated force expresses only the hydraulic force generated by the shock absorber.

In all graphical results presented, positive displacements, velocities and forces denote rebound of the shock absorber, while negative displacements, velocities and forces denote compression of the shock absorber.

Figure 7 (a), and 7(b) shows the displacement-time, velocity-time and force-time characteristics of the shock absorber at $1 \mathrm{~Hz}$ and for 20 and $50 \mathrm{~mm}$ amplitude respectively. Both figures show that the displacement and velocity input excitation signals are sinusoidal. However, the figures show that the output force response is approximately sinusoidal in the compression and rebound strokes. The figures show 


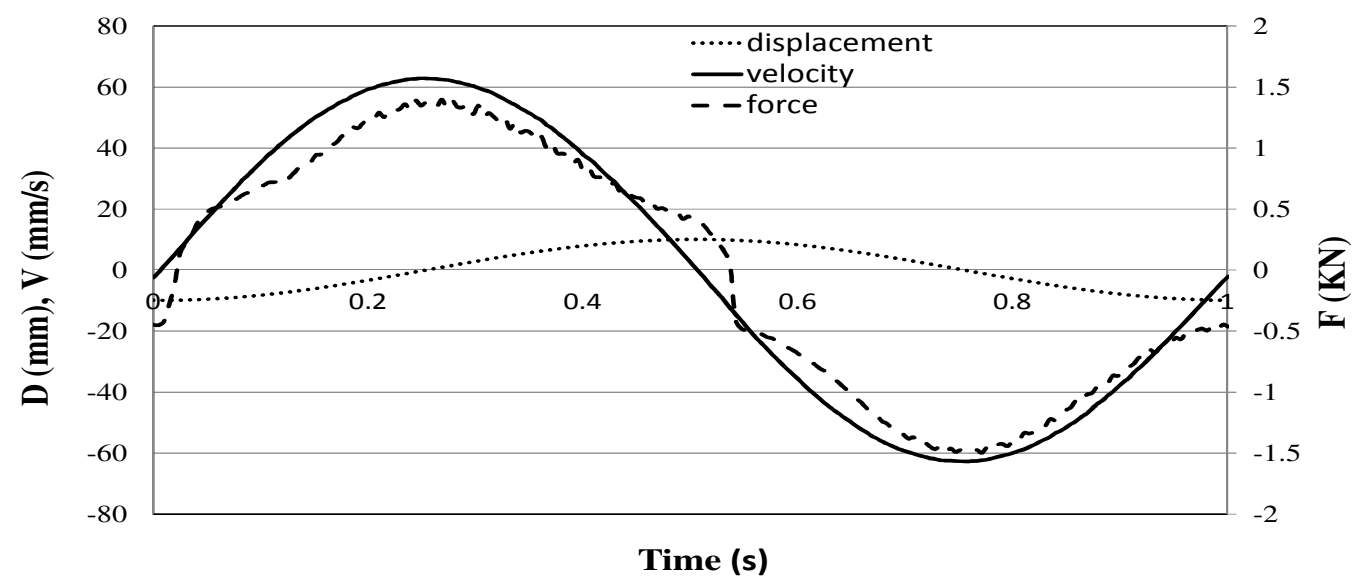

(a)

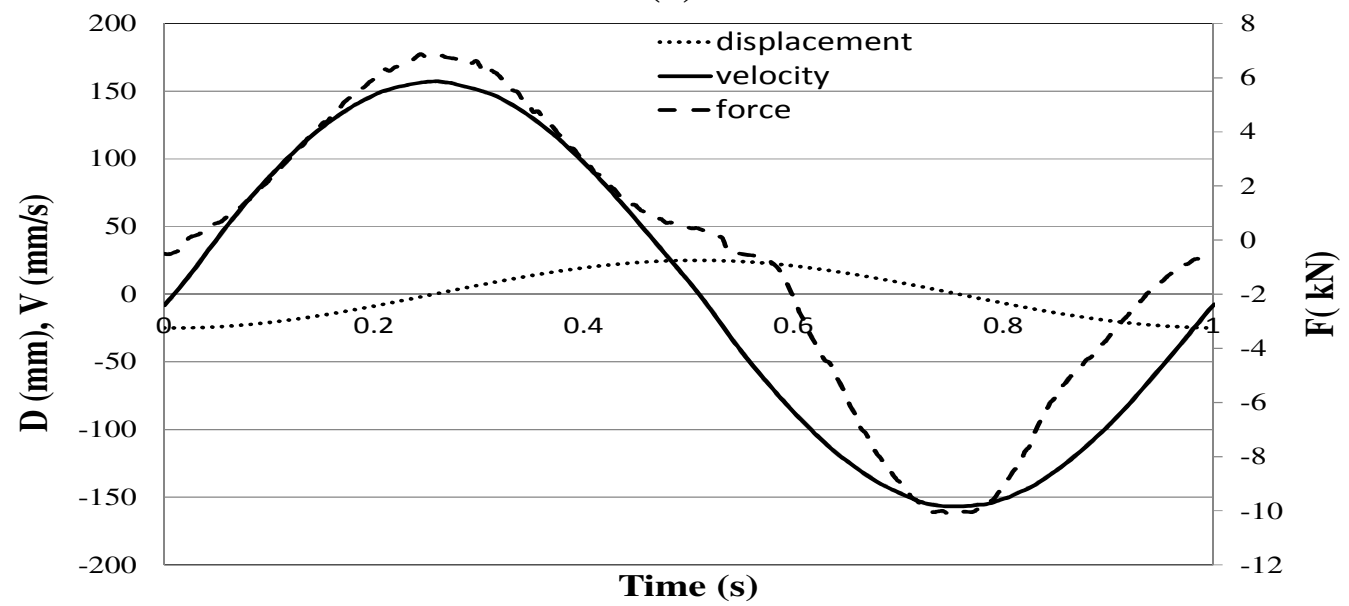

(b)

Figure 7: Displacement, velocity and force of shock absorber at $1 \mathrm{~Hz}$ excitation frequency and for (a) $20 \mathrm{~mm}$ and (b) $50 \mathrm{~mm}$ amplitudes.

that the peak force in the rebound stroke is lower than that of the compression stroke. Consequently, the shock absorber is relatively hard in compression but relatively soft in rebound.

Figure 8 shows the variation of the damping force with piston displacement for sine wave excitation with $1 \mathrm{~Hz}$ frequency and with different amplitudes from $10-50 \mathrm{~mm}$ while figure 9 shows the variation of the damping force with piston velocity at the same excitation conditions. High damping force is obtained in compression stroke compared with the rebound stroke for all values of excitation amplitudes. The damping force produced by the shock absorber, ranges from about -10 to $7 \mathrm{kN}$.

The previous analysis shows that at low frequencies of excitation, the hydraulic shock absorber reveals higher damping force in compression stroke than in rebound, which implies that the shock absorber is soft in rebound but hard in compression.

Figures 10,11, and 12 illustrate the variation of peak damping force with peak velocity for different amplitudes of excitation from (10-50) $\mathrm{mm}$ at excitation frequency of 1,4 and $5 \mathrm{~Hz}$ respectively. It is shown from the figures that as the frequency of excitation 


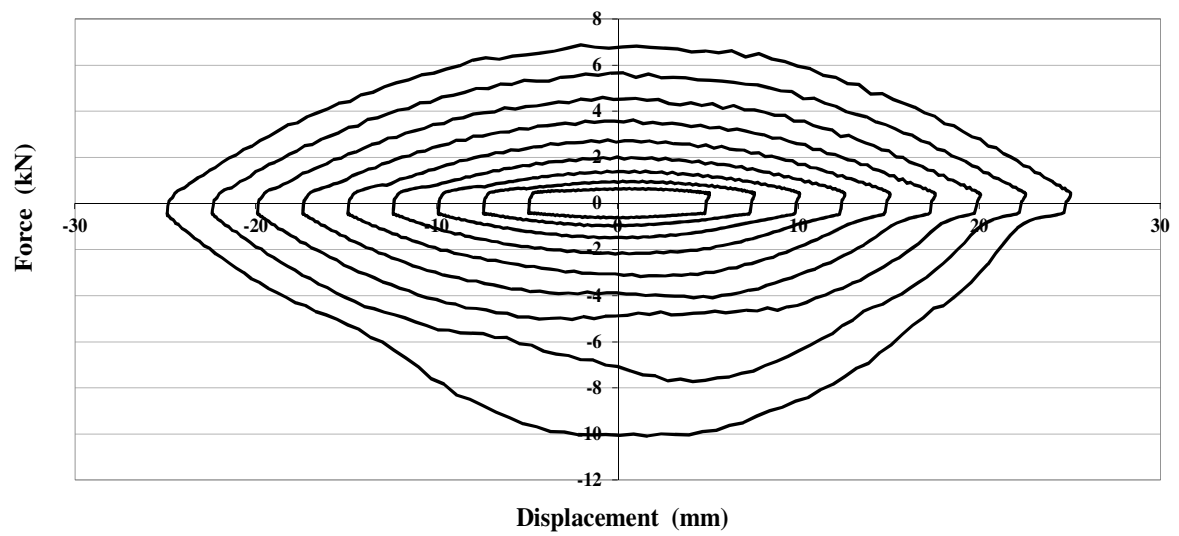

Figure 8: Force - displacement characteristics at $1 \mathrm{~Hz}$ and (10-50) mm.

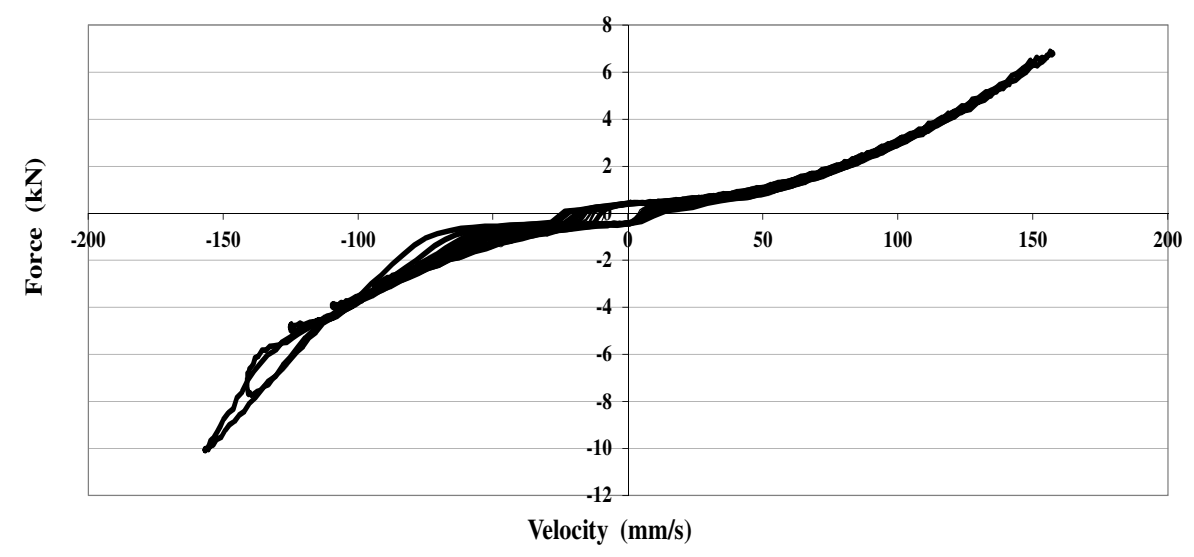

Figure 9: Force -velocity characteristics at $1 \mathrm{~Hz}$ and (10-50) $\mathrm{mm}$.

increases the peak force in compression and rebound strokes increases. The rebound stroke reveals more damping force than in compression stroke at high frequencies, which implies that the damper is soft in compression than in rebound. Otherwise, at low frequency the damping force in compression is slightly higher than in rebound, which means that damper is soft in rebound than in compression. It is shown also, that as the frequency of excitation increases, the piston velocity in rebound stroke decreases, which confirm the fact that in automotive vehicles the damper in rebound moves slowly than in compression and preserve more damping force.

The relation between damping force and velocity shown in figure 13 is used to obtain the damping coefficient. The graph represents the trend line of the real results measured at excitation frequency of $3 \mathrm{~Hz}$ as an intermediate frequency of the measured range. Thus the obtained value of the damping coefficient represents the average value used during obtaining the vibrational response of the vehicle hull.

\section{RESULTS}

The vibrational response of the BMP-1 in pitch and bounce obtained from the Simulink model in case of excitation by sinusoidal and random roads are discussed hereafter. 


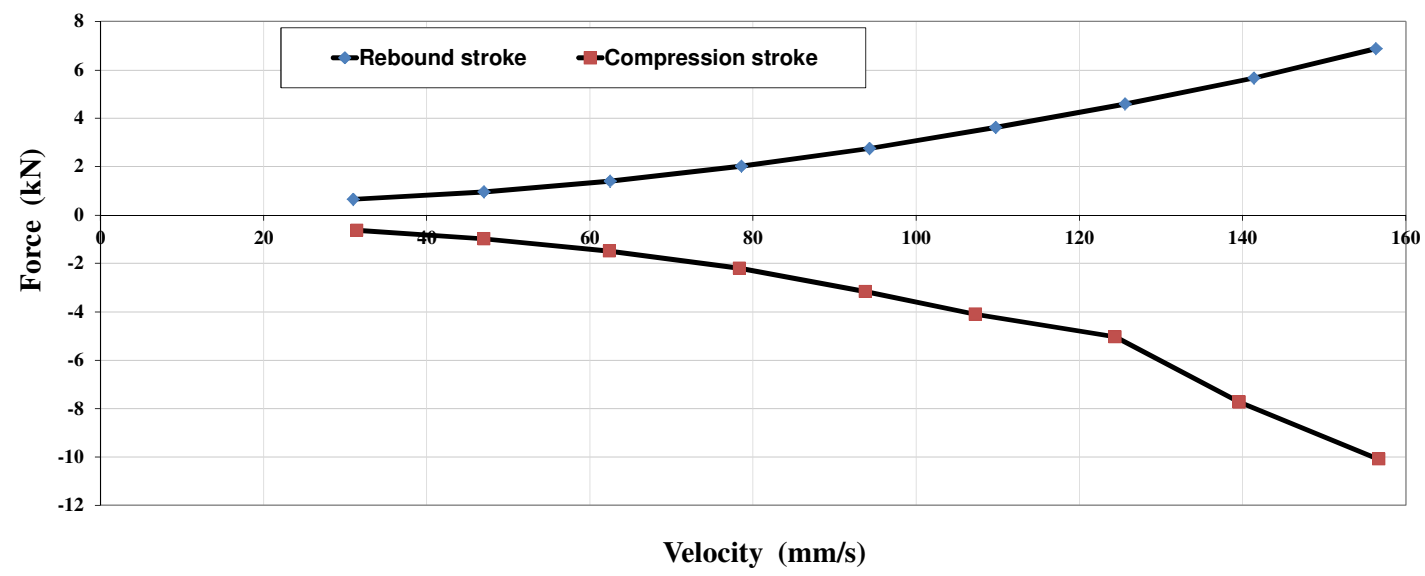

Figure 10: The peak force -peak velocity at (1) $\mathrm{Hz}$ and (10-50) mm.

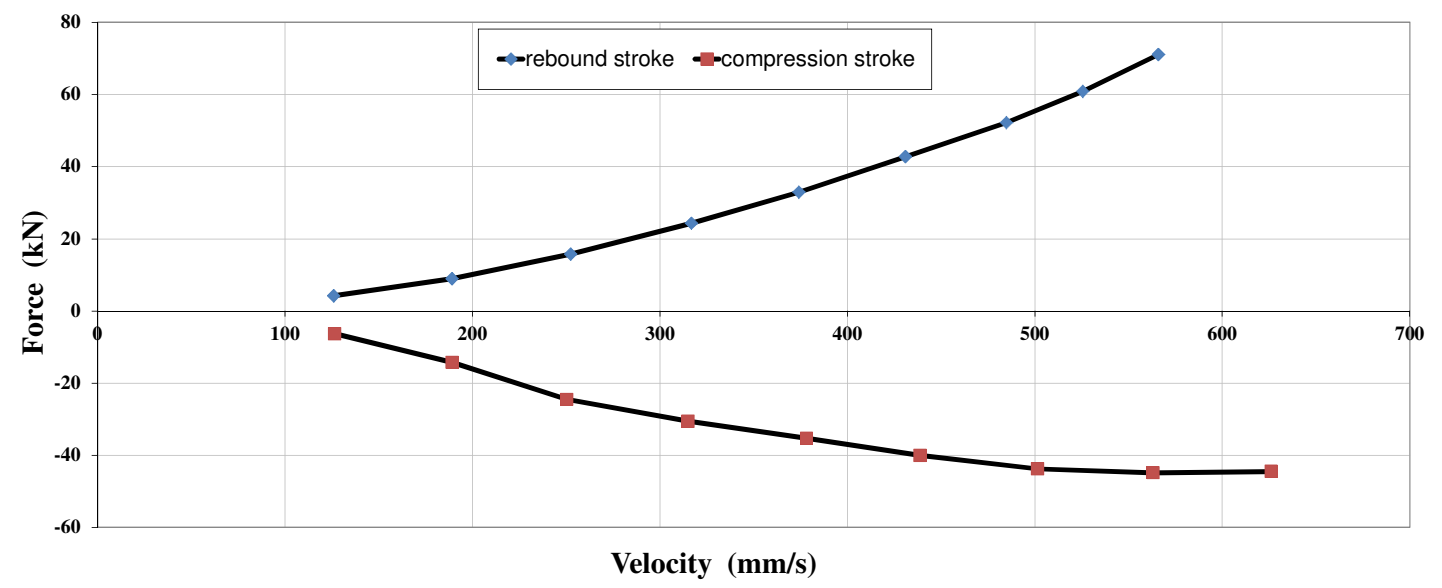

Figure 11: The peak force - peak velocity at (4) $\mathrm{Hz}$ and (10-50) $\mathrm{mm}$.

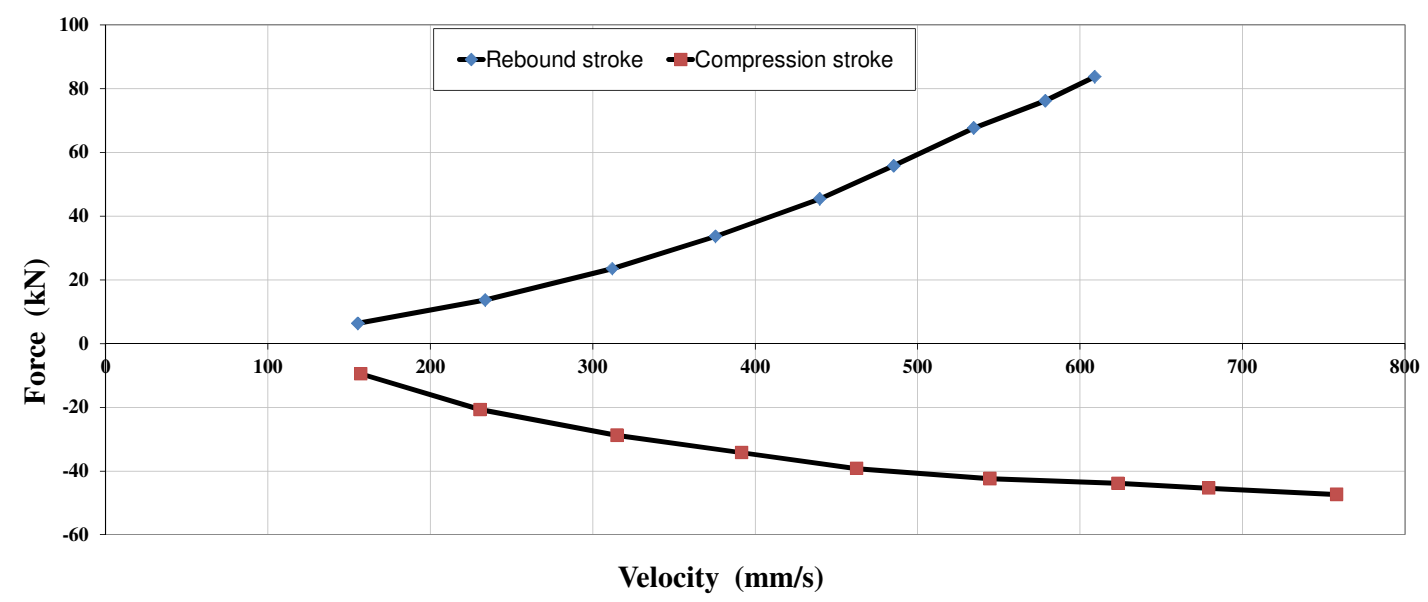

Figure 12: The peak force - peak velocity at (5) $\mathrm{Hz}$ and (10-50) mm. 


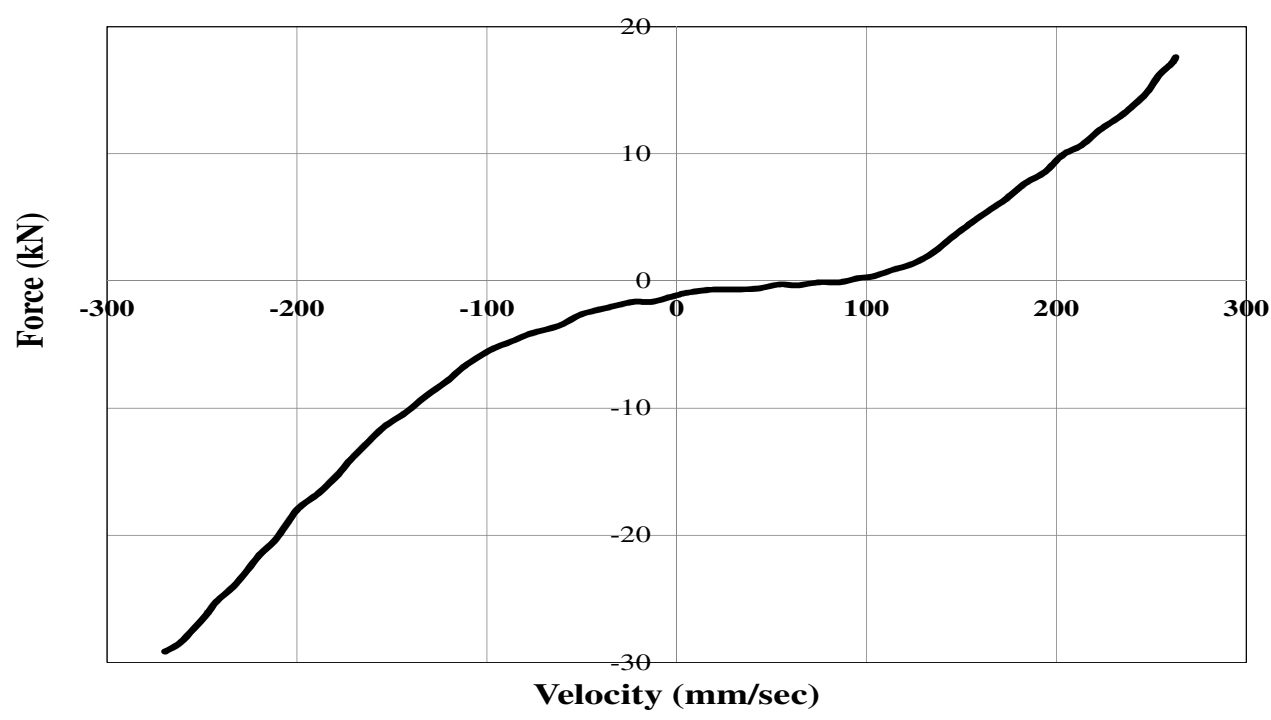

Figure 13: Average characteristics of BMP-1 shock absorber.

\section{Sine Wave Oscillation}

Figures 14 (a), 14 (b) show the angular and vertical displacement of vehicle hull in case of excitation with sinusoidal road respectively. The figure confirms the sinusoidal excitation of the road. As observed from the figure, the response of vehicle hull is composed of 2 oscillations: a damped free oscillation at a damped frequency and a forced oscillation at the excitation frequency. The damped free oscillation disappears soon and remains only the forced oscillation in the steady state.

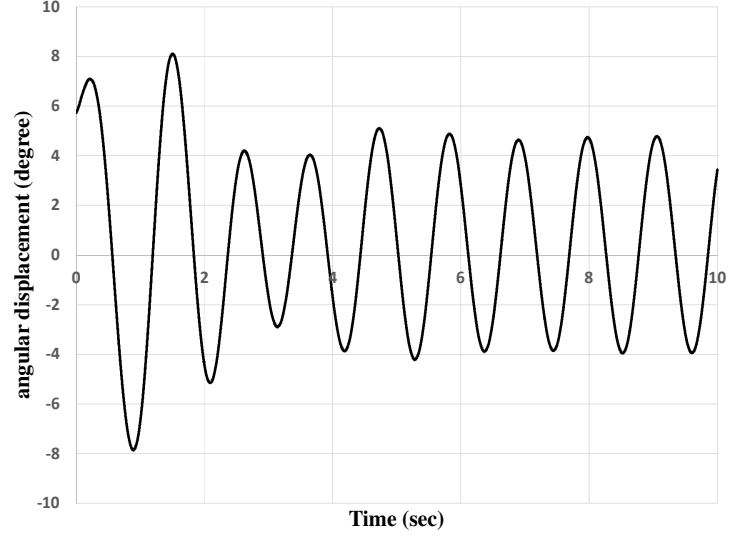

(a)

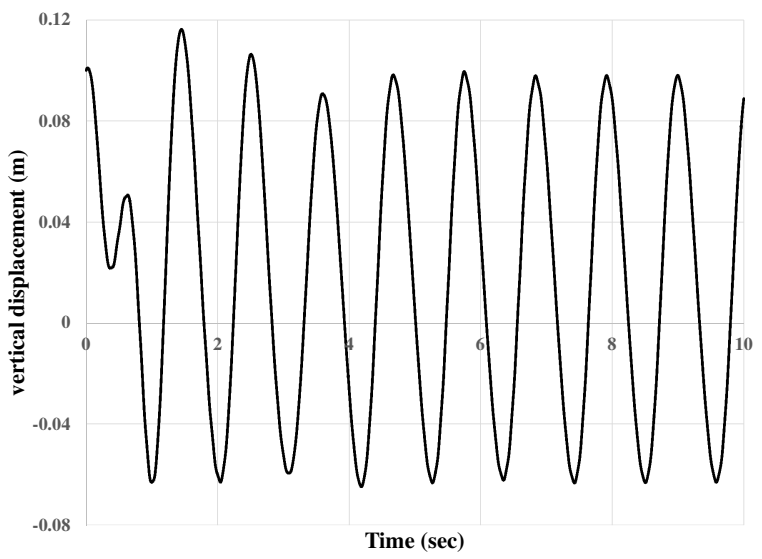

(b)

Figure 14: vehicle response due to sinusoidal road excitation

(a) angular displacement, (b) vertical displacement.

\section{Random Wave Oscillation}

Figures 15 (a), 15(b) demonstrate the angular displacement and vertical displacement of vehicle hull respectively in case of excitation with random road profile. The figures 
demonstrate random responses of the vehicle when it is running on the rough surface, previously defined, with the vehicle speed of $20 \mathrm{~km} / \mathrm{h}$.

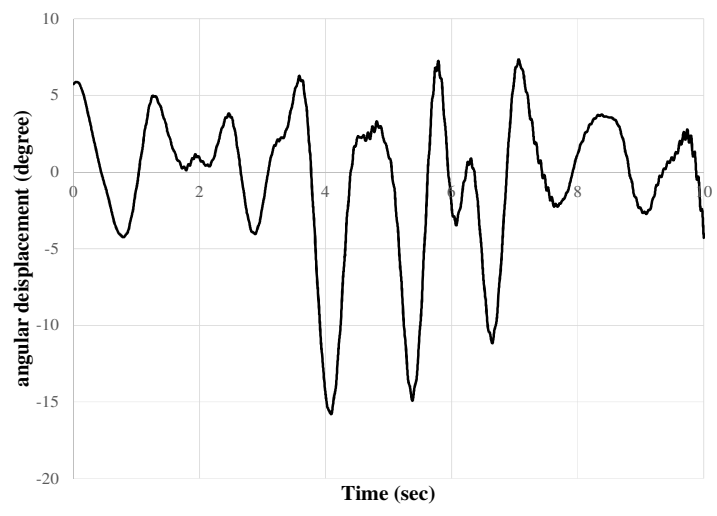

(a)

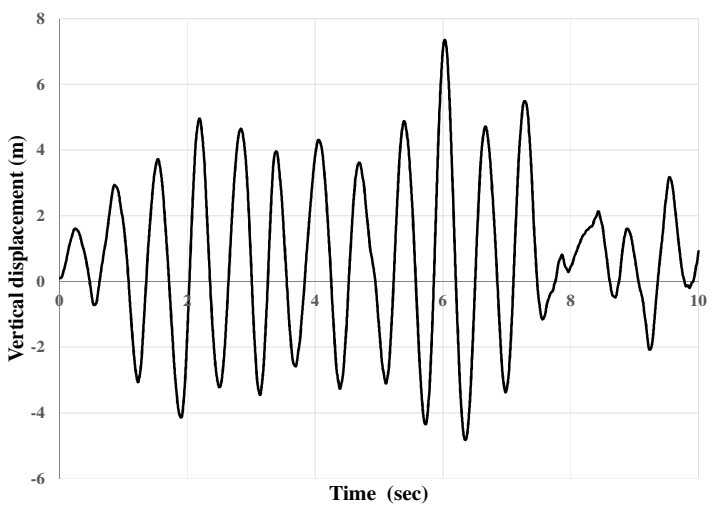

(b)

Figure 15: Vehicle response due to random road excitation

(a) angular displacement, (b) vertical displacement.

\section{CONCLUSION}

- The suspension system characteristics of BMP-1 tracked vehicle are evaluated and the variation of suspension stiffness with road wheel displacement is found to have a nonlinear characteristics.

- The operational characteristics of the shock absorber of BMP-1 tracked vehicle are evaluated using Damper Test System where it is found that the hydraulic shock absorber has nonlinear characteristics in both rebound and compression strokes. At low frequencies $(1,2) \mathrm{Hz}$, the damping force in compression is higher than that in rebound while above $2 \mathrm{~Hz}$, the damping in rebound is higher than in compression. It is found also that with increasing either the frequency or the amplitude of excitation, the damping force increases intensely.

- A Matlab/Simulink suspension model of a half vehicle is developed. The model has 8 DOF including the response of the bounce and pitch for vehicle hull and the vertical displacement of six road wheels. The vibrational response of the BMP-1 is evaluated in pitch and bounce for sinusoidal and random road profiles using the real characteristics of suspension parameters (suspension and shock absorber characteristics). The results show that the vehicle response follow the road shape as for sinusoidal road excitation the hull performance has a sinusoidal response and the same criteria is obtained for the random road.

\section{REFERENCES}

[1] D. Kowalski, M. D. Rao, J. Blough, S. Gruenberg, and D. Griffiths, "The effects of different input extcitation on the dynamic characterization of automotive shock absorbers," Michigan Technological University, 2001. 
[2] W. Schiehlen and B. Hu, "Spectral simulation and shock absorber identification," International Journal of Non-Linear Mechanics, vol. 38, pp. 161$171,2003$.

[3] S. W. R. Duym, "Simulation tools, modelling and identification, for an automotive shock absorber in the context of vehicle dynamics," Vehicle System Dynamics, vol. 33, pp. 261-285, 2000.

[4] J. N. Yang, J.-H. Kim, and A. K. Agrawal, "Resetting semiactive stiffness damper for seismic response control," Journal of Structural Engineering, vol. 126, pp. 1427-1433, 2000.

[5] Oyadiji S. O. and Sarafianos P., "Characterisation and comparison of the dynamic properties of conventional and electro-rheological fluid shock absorbers," International Journal of Vehicle Design, vol. 33, pp. 251-278, 2003.

[6] R. H. Khodary, M. Ragheb, A.M.Salem, A.M.Zidan, "Vibrational Response of Tracked Vehicles with Variable Suspension Characteristics," 14th int.AMME conference 25-27 May 2010, Cairo, Egypt, 2010.

[7] Y. Yu, X.-x. Wei, and Y.-f. Zhang, "Fuzzy logic control for suspension systems of tracked vehicles," Journal of Beijing Institute of Technology, vol. 18, pp. 3740, 2009.

[8] U. Solomon and C. Padmanabhan, "Hydro-gas suspension system for a tracked vehicle: Modeling and analysis," Journal of Terramechanics, vol. 48, pp. 125137, 2011.

[9] P. J. Liu, L. Hou, W. G. Lin, X. Y. Yu, and W. Huang, "Simulation of Ride Comfort of Tracked Vehicle Based on Road Random Excitation," Advanced Materials Research, vol. 479, pp. 93-97, 2012.

[10] J. Y. Wong and J. Preston-Thomas, "Investigation into the effects of suspension characteristics and design parameters on the performance of tracked vehicles using an advanced computer simulation model," Proceedings of the Institution of Mechanical Engineers, Part D: Journal of Automobile Engineering, vol. 202, pp. 143-161, 1988.

[11] B. Maclaurin, "Progress in British tracked vehicle suspension systems," SAE Technical Paper 0148-7191, 1983.

[12] Choi, S. B., Suh, M. S., Park, D. W., Shin, and M. J., "Neuro-fuzzy control of a tracked vehicle featuring semi-active electro-rheological suspension units," Vehicle System Dynamics, vol. 35, pp. 141-162, 2001. 